\title{
Covid-19 : Analyse spatiale de la létalité hospitalière en France durant la première vague épidémique
}

Marc Souris et Jean-Paul Gonzalez

\section{CpenEdition}

Journals

Édition électronique

URL : http://journals.openedition.org/rfst/894

DOI : $10.4000 /$ rfst.894

ISSN : 2492-3672

Éditeur

Espaces et SOciétés (UMR 6590)

Référence électronique

Marc Souris et Jean-Paul Gonzalez, «Covid-19: Analyse spatiale de la létalité hospitalière en France durant la première vague épidémique », Revue francophone sur la santé et les territoires [En ligne], Pandémie, crises et perspectives : lectures territoriales de la Covid-19, mis en ligne le 11 mars 2021, consulté le 06 avril 2021. URL : http://journals.openedition.org/rfst/894 ; DOI : https://doi.org/ $10.4000 /$ rfst.894

Ce document a été généré automatiquement le 6 avril 2021.

\section{cc) (i)}

La Revue francophone sur la santé et les territoires est mise à disposition selon les termes de la Licence Creative Commons Attribution - Pas d'Utilisation Commerciale - Partage dans les Mêmes Conditions 4.0 International. 


\title{
Covid-19 : Analyse spatiale de la létalité hospitalière en France durant la première vague épidémique
}

\author{
Marc Souris et Jean-Paul Gonzalez
}

\section{Introduction}

1 Le taux de létalité réel est le rapport entre le nombre de décès dus à la maladie et le nombre de personnes infectées (guéries ou décédées). Il est estimé par le système de santé à partir de la déclaration de ces deux valeurs. Le taux de létalité est en principe indépendant de l'étendue de la maladie, contrairement aux taux de mortalité ou de morbidité (Porta M., 2008).

2 Les taux de létalité de la Covid-19 publiés au premier semestre 2020 dans les médias varient considérablement entre les pays, ce qui engendre de nombreuses interrogations sur la susceptibilité de la population, sur les politiques nationales de réponse à la pandémie, sur les systèmes de déclaration des cas, sur les protocoles de prise en charge des patients. Cette question de la létalité de la Covid-19 a été abordée dès le début de l'épidémie (Morteza \& Fakher, 2020 ; Rinaldia \& Paradisiba, 2020 ; Spychalski \& Blazynska-Spychalska, 2020 ;Baud \& Qi, 2020.

3 La létalité dépend de plusieurs types de facteurs : de facteurs liés au virus lui-même (ex. virulence, dose infectante, mutation), de facteurs liés à la population(risques biologiques, vulnérabilités - i.e. âge, facteurs génétiques, statut immunitaire, comorbidités, accès aux soins., etc.), et de facteurs directement liés au système de santé (ex. équipement, capacité d'accueil, personnel, gestion, prise en charge des malades, efficacité des thérapies, prise en charge en phase critique de la maladie), et de facteurs liés au système de détection et d'enregistrement des cas et des décès (définition du cas clinique, détection, dénombrement et déclaration des cas et des décès, systèmes de 
surveillance, saisie des données). L'estimation de la létalité nécessite de détecter et dénombrer tous les infectés, quel qu'en soit le tableau clinique. Toutefois ce nombre d'infectés est difficile à évaluer du fait de la variabilité de la clinique ou de la présence de forme asymptomatiques.

4 Au 8 mai 2020, il existait de fortes différences dans les taux de létalité de la Covid-19 publiés par pays (Tableau 1) ou calculés à partir des données de l'OMS. Les causes de ces différences peuvent provenir de l'ensemble des facteurs mentionnés plus haut. La virulence peut évoluer dans le temps et dans l'espace si un risque de mutation/ sélection naturelle du pathogène existe (Collier \& Oxford, 2016 ; Saluzzo \& Vidal, 2004 ; Hurraux \& Nicolas, 2003), ces mutations n'ayant pas été observées durant la période étudiée la virulence du virus SARS-CoV-2 sera supposée identique durant cette période. Par contre, tous les autres facteurs peuvent présenter des variations selon les pays, et notamment celles liés à la définition, la détection, la déclaration des cas et des causes du décès.

Tableau 1 : Taux de létalité et facteurs de vulnérabilité de la population à la Covid-19

\begin{tabular}{|c|c|c|c|}
\hline Pays & $\begin{array}{l}\text { Taux de létalité } \\
(\%)^{\star}\end{array}$ & $\begin{array}{c}\text { Population }>65 \text { ans } \\
(\%)^{\star \star}\end{array}$ & $\begin{array}{l}\text { Capacité hospitalière } \\
\text { (nombre de lits pour } \\
100000 \text { personnes) }\end{array}$ \\
\hline France & 19.00 & 20.02 & 621 \\
\hline Belgium & 16.42 & 18.80 & 623 \\
\hline UK & 14.94 & 18.40 & 273 \\
\hline Italx & 13.84 & 22.75 & 331 \\
\hline Netherlands & 12.59 & 19.20 & 466 \\
\hline Sweden & 12.29 & 20.10 & 254 \\
\hline Spain & 11.73 & 19.38 & 297 \\
\hline Iran & 6.31 & 6.18 & 150 \\
\hline USA & 5.40 & 15.80 & 290 \\
\hline Greece & 5.52 & 21.65 & 424 \\
\hline China & 5.50 & 10.92 & 420 \\
\hline Switzerland & 5.01 & 18.62 & 470 \\
\hline Germany & 4.28 & 21.46 & 823 \\
\hline Portugal & 4.15 & 21.95 & 332 \\
\hline Austria & 3.88 & 19.02 & 760 \\
\hline Tanzania & 3.75 & 2.60 & 70 \\
\hline Japan & 3.56 & 27.58 & 1340 \\
\hline \begin{tabular}{l|} 
India \\
\end{tabular} & 3.36 & 6.18 & 70 \\
\hline Szzech Republic & 3.28 & 19.42 & 645 \\
\hline Turkey & 2.72 & 8.48 & 270 \\
\hline Norway & 2.62 & 17.05 & 390 \\
\hline Uruguay & 2.53 & 14.81 & 280 \\
\hline South Korea & 2.36 & 14.42 & 1150 \\
\hline Thailand & 1.83 & 11.90 & 210 \\
\hline Slovakia & 1.74 & 15.63 & 579 \\
\hline Australia & 1.41 & 15.65 & 380 \\
\hline Chile & 1.21 & 11.53 & 220 \\
\hline
\end{tabular}

Légende : *Taux de létalité pour la Covid-19 pour quelques pays, à partir des données de l'OMS au 8 mai 2020 (https://covid19.who.int/) ; **pourcentage de population âgée de plus de 65 ans, Banque Mondiale 2016; ***Nombre de lits d'hôpitaux pour 100000 personnes, Banque Mondiale, 2013, Eurostat, 2014.

5 De ces observations plusieurs questions/hypothèses ont émergées: Trouve-t-on également des variations spatiales significatives de la létalité hospitalière au sein d'un même pays, et de quel ordre? Quelles sont les causes de ces variations, alors que la définition et la déclaration des cas sont supposées constantes en France ? L'augmentation de la létalité est-elle liée à la tension hospitalière ?

6 L'objet de cet article est, pour tenter de répondre à ces questions, d'analyser la distribution spatiale de la létalité de la Covid-19 à l'échelle des départements en France 
métropolitaine, pendant la première vague de l'épidémie du 19 mars au 28 mai 2020. L'analyse se focalisera sur les causes liées au système de soins grâce à l'élimination par standardisation des facteurs de risques liés à l'âge de la population.

\section{Données et méthodes}

\section{Données}

7 Cette étude est basée sur les données de déclaration journalière d'hospitalisation et de décès par département et accessibles sur le site de Santé Publique France à partir du 19 mars 2020, ainsi que des données de distribution des cas hospitalisés en fonction de la classe d'âge (par tranche de dix ans, Santé Publique France, www.data.gouv.fr/fr/ datasets/donnees-hospitalieres-relatives-a-lepidemie-de-Covid-19).

8 Nous disposions également de plusieurs jeux de données: démographiques par département (population par âge, INSEE, 2020); état de santé de la population (affections de longue durée pour les diabètes, les affections cardiovasculaires, l'obésité, l'hypertension) et système de soins hospitalier par département (nombre de lits d'hôpital) (Ecosanté France, www.ecosanté.fr, 2016) ; accessibilité aux soins libéraux (APL, indicateur d'accessibilité spatiale localisée, www.data.drees.sante.gouv.fr).

Cette analyse a été effectuée sur les 96 départements de France métropolitaine. Les départements et territoires français d'outre-mer ont été exclus de l'analyse spatiale pour des raisons de contiguïté spatiale (notamment pour l'analyse de l'autocorrélation spatiale, qui est liée à la distance entre départements). Les données ont été intégrées dans un système d'information géographique (SavGIS) afin de les analyser et de les cartographier.

\section{Méthodes}

Les taux de morbidité ou de létalité calculés dans cet article portent uniquement sur les cas hospitalisés et sur les décès à l'hôpital liés à la Covid-19 (testés positifs par RT-PCR).

11 Ces taux ne représentent pas la morbidité et létalité réelle (qui devrait inclure tous les cas infectés et tous les décès liés à la Covid-19), ni la létalité observée (qui inclue tous les cas positifs confirmés, même asymptomatiques), mais le taux de morbidité hospitalière (rapport entre nombre de cas hospitalisés et effectif de population, appelé dans la suite taux d'hospitalisation) et la létalité hospitalière (rapport entre le nombre de décès chez les cas hospitalisés et le nombre de ces cas hospitalisés). Compte-tenu du nombre estimé élevé de formes bénignes ou asymptomatiques (Salie \& Tran-Kiem , 2020), la létalité réelle (tout comme la létalité observée) est très inférieure à la létalité hospitalière, mais ne pourra être calculée avec précision que lorsque pourra être estimé le nombre total de personnes infectés ainsi que le nombre total de décès (y compris hors hôpital) dus à la Covid-19.

12 Il est supposé que le système de définition et dénombrement des cas hospitalisés et des décès à l'hôpital a été identique sur l'ensemble du territoire métropolitain français durant la période étudiée, et donc que les différences de létalité internes à la France sont indépendantes de ce système de dénombrement. 


\section{Standardisation sur l'âge}

13 Pour comparer les taux de morbidité ou de létalité de plusieurs régions indépendamment des caractéristiques de la population, il est nécessaire, dans le calcul des taux, de standardiser les facteurs de risque liés à la population (Bouyer \& Hémon, 1993).

L'âge étant un facteur de risque majeur d'hospitalisation ou de décès pour les patients Covid-19, il est ainsi important d'éliminer les différences entre départements dues aux différences de structure d'âge de la population. Nous avons donc effectué une standardisation indirecte sur l'âge en calculant les taux (d'hospitalisation et de létalité hospitalière) par classe d'âge de dix ans sur l'ensemble de la France. Ces taux ont également été estimés en excluant les zones qui ont été en tension hospitalière (Ile-deFrance et Grand-Est) durant cette première vague, afin d'évaluer les taux de létalité hospitalière par classe d'âge indépendamment d'une surmortalité éventuelle due à la tension hospitalière de certains départements.

\section{Ratio et taux standardisés de morbidité et de létalité}

De façon classique, l'effectif $\boldsymbol{E}_{\boldsymbol{i}}$ (de malades ou de décès) attendus dans le département $\boldsymbol{i}$ est la somme, sur les différentes classes d'âge, de la population $\boldsymbol{P}_{i, a}$ du département $\boldsymbol{i}$ dans la classe d'âge $\boldsymbol{a}$ multiplié par $\boldsymbol{T}_{\boldsymbol{a}}$, le taux (de morbidité ou de létalité) hospitalière de la classe d'âge $\boldsymbol{a}$ calculé sur l'ensemble du pays :

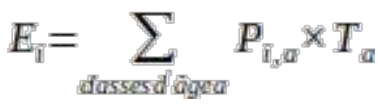

Le ratio standardisé (de morbidité, SMR, pour Standardized Morbidity Ratio, et de létalité, SLR, pour Standardized Lethality Ratio est égal au rapport entre l'effectif observé $\boldsymbol{O}_{i}$ et l'effectif attendu $E_{i}$. Le taux de létalité standardisé d'un département $T L S_{i}$ est égal au taux de létalité moyen sur la France TL multiplié par le SLR du département :

$$
T L S_{\mathrm{i}}=T L \times S L R_{\mathrm{i}}
$$

17 L'évaluation de la significativité des ratio standardisés (par exemple H0 : SLR=1, pas de sur- ou sous-létalité statistiquement significative) est évaluée pour chaque département par un test de Breslow \& Day (Breslow \& Day, 1987) afin de rendre compte de la significativité statistique des différences de ce ratio entre les départements. Afin de tenir compte du problème des tests multiples (Miller, 1996) lorsque l'hypothèse nulle concerne l'ensemble du territoire ( $\mathrm{y}$ a-t-il un département pour lequel l'hypothèse $\mathrm{H0}$ est rejetée ? »), le risque d'erreur a été fixé à 0.0001 afin d'appliquer une correction de Bonferonni (très conservatrice ici puisque les taux présentent de l'autocorrélation spatiale). Lorsque l'étude porte sur un seul département (« Le SLR de ce département est-il différent de 1 ? »), le risque d'erreur est fixé à 0.05 . Tous les tests sont bilatéraux.

\section{Étude des corrélations statistiques}

18 Afin d'estimer si la tension hospitalière a influencé le taux de létalité hospitalière, nous avons étudié les corrélations entre hospitalisation et létalité hospitalière par département. La sévérité a été estimée en utilisant le taux de réanimation (nombre de patients admis en soins intensifs réanimation et/ou intubation endotrachéale divisée 
par le nombre de patients hospitalisés). Nous avons également analysé la relation entre le taux de létalité standardisé et la capacité du système de santé (i.e. nombre de lits par personne).

Les analyses statistiques et spatiales, ainsi que les représentations cartographiques ont été effectuées grâce au système d'information géographique SavGIS (Souris, 2019).

\section{Résultats}

L'épidémie de Covid-19 en France a connu un premier pic puis une période de moindre circulation du virus après un premier confinement du 22 mars au 11 mai (Fig. 1). Notre étude porte sur la première vague, du 19 mars (date du début de la disponibilité des données sur le site de Santé Publique France), au 28 mai.

Fig. 1. Hospitalisations (à gauche), décès (à droite). Moyenne mobile sur 7 jours, du 19 mars au 28 mai 2020
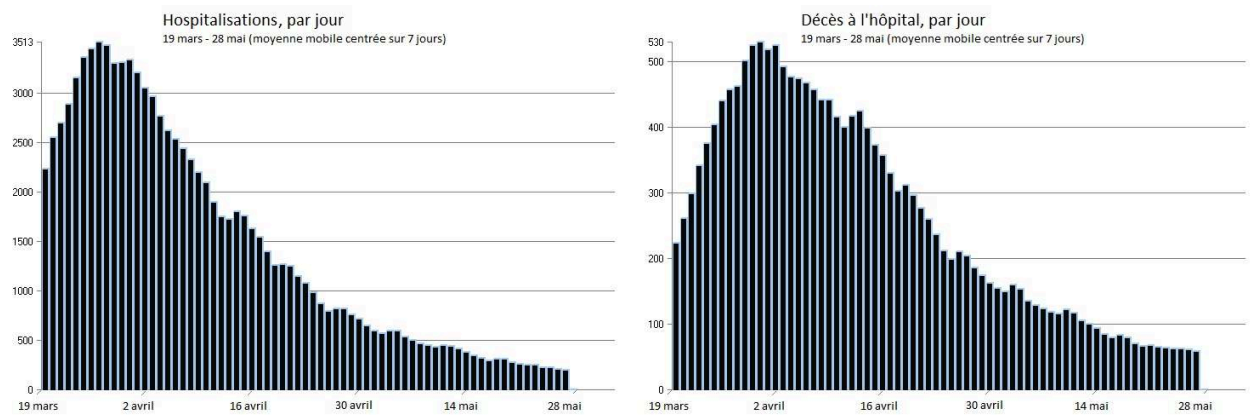

Sur l'ensemble de la France métropolitaine du 19 mars au 28 mai, le taux de létalité hospitalière s'élève à 0.2143 .

\section{Analyse temporelle}

Le taux de létalité a fortement évolué au cours de la période étudiée (tableau 2).

Tableau 2 : Taux de létalité Covid-19 en France métropolitaine du 19 mars au 28 mai. Hospitalisations, retour à domicile (RAD), décès (DCD), létalité, par période de 14 jours, du 18 mars au 28 mai 2020

\begin{tabular}{|c|c|c|c|c|c|c|}
\hline & Au 18 mars & 19 mars -1 avril & 2 avril - 15 avril & 16 avril-29 avril & 30 avril-13 mai & $\begin{array}{c}14 \text { mai - 27 } \\
\text { mai }\end{array}$ \\
\hline HosR. & 2972 & 24543 & 31623 & 26736 & 20961 & 15627 \\
\hline RAD & 816 & 10118 & 20018 & 17269 & 10443 & 7911 \\
\hline DCD & 218 & 3814 & 6611 & 4398 & 2041 & 1158 \\
\hline Létalité & 0.211 & 0.274 & 0.248 & 0.203 & 0.163 & 0.128 \\
\hline
\end{tabular}

L'analyse temporelle montre que le niveau d'hospitalisation a évolué à la baisse dans toutes les régions avec la même dynamique, mais par contre le taux de létalité a évolué de façon différente en fonction des régions et de leur niveau d'hospitalisation (exemple des régions Ile-de-France et Aquitaine, Fig. 2). Toutefois, ces résultats sont limités par la difficulté de calculer un taux de létalité sur une courte période à partir de données agrégées, la durée d'hospitalisation n'étant pas la même pour les décès et les retours à domicile (Salie \& Tran-Kiem, 2020 ; Courtejoie \& Dubost, 2020). 
Fig. 2. Hospitalisations et létalité en régions lle-de-France (en haut) et Aquitaine (en bas). Moyenne journalière mobile sur 7 jours, du 19 mars au 28 mai 2020
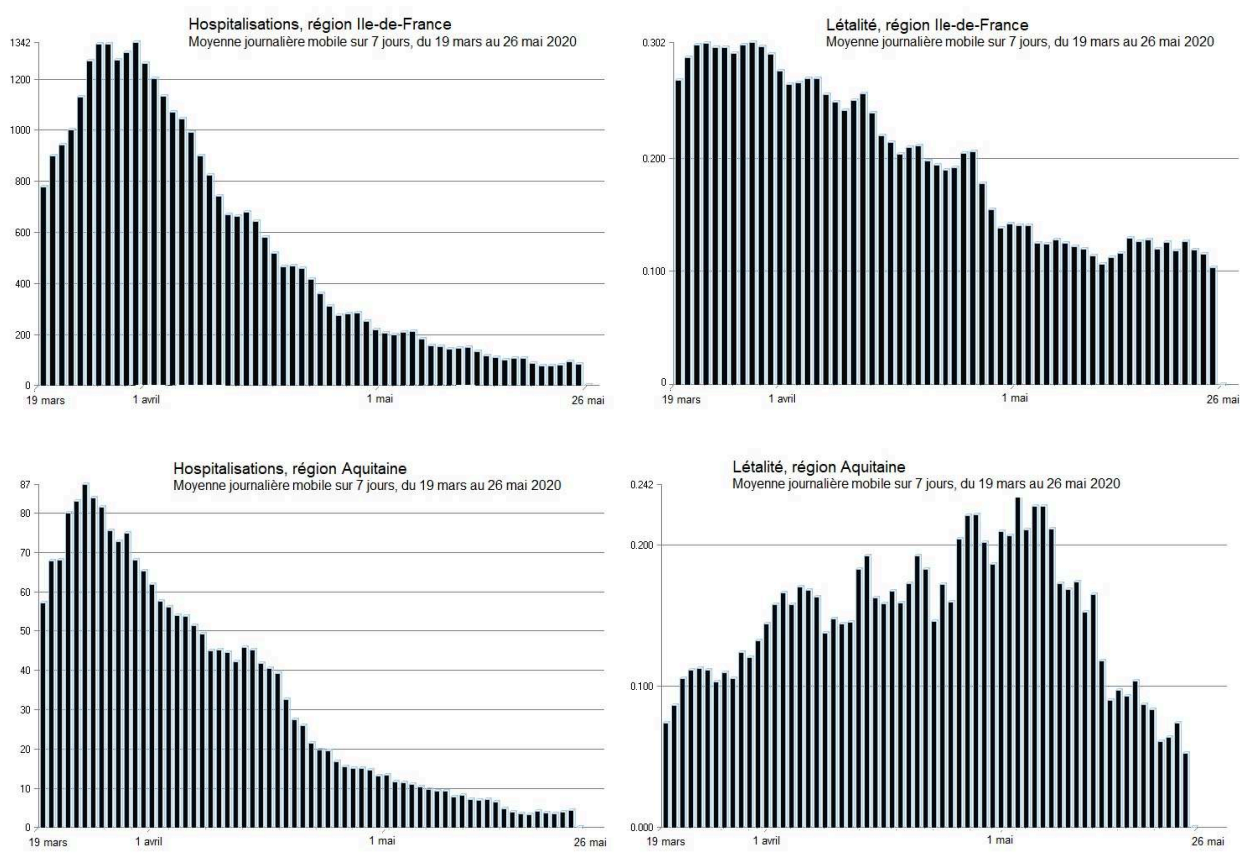

Les taux de létalité hospitalière par classe d'âge sur l'ensemble de la France métropolitaine, utilisés pour la standardisation indirecte, ont été calculés pour les cas fermés (c'est-à-dire dont on connait l'issue, décès ou retour à domicile) à partir des données par classe d'âge de Santé Publique France (Tableau 3).

Tableau 3. Hospitalisation, mortalité, et létalité par classe d'âge en France métropolitaine du 19 mars au 28 mai 2020 (Santé Publique France)

\begin{tabular}{|c|c|c|c|c|c|c|c|}
\hline \multirow[b]{3}{*}{$\begin{array}{l}\text { Classe } \\
\text { d'âge }\end{array}$} & \multirow[b]{3}{*}{$\begin{array}{l}\text { Population } \\
\text { (\%) }\end{array}$} & \multicolumn{6}{|c|}{ Hospitalisations (19 mars-28 mai 2020$)$} \\
\hline & & \multicolumn{2}{|c|}{ Hospitalisés } & \multicolumn{2}{|c|}{ Décès } & \multicolumn{2}{|c|}{ Létalité } \\
\hline & & Total & $\begin{array}{l}\% \text { du } \\
\text { total }\end{array}$ & Total & $\begin{array}{l}\% \text { du } \\
\text { total }\end{array}$ & $\begin{array}{l}\text { France } \\
\text { métro. }\end{array}$ & $\begin{array}{l}\text { Sans les } \\
\text { régions lle- } \\
\text { de-France et } \\
\text { Grand-Est }\end{array}$ \\
\hline $0-9$ & 11.43 & 682 & 0.68 & 3 & 0.01 & 0.0046 & 0 \\
\hline $10-19$ & 12.27 & 509 & 0.51 & 3 & 0.01 & 0.0064 & 0 \\
\hline $20-29$ & 11.15 & 2388 & 2.39 & 21 & 0.12 & 0.0093 & 0.0058 \\
\hline $30-39$ & 12.36 & 4556 & 4.56 & 82 & 0.45 & 0.0193 & 0.0096 \\
\hline $40-49$ & 12.80 & 7314 & 7.32 & 229 & 1.26 & 0.0339 & 0.0204 \\
\hline $50-59$ & 13.10 & 12648 & 12.65 & 850 & 4.67 & 0.0755 & 0.0523 \\
\hline $60-69$ & 12.01 & 16861 & 16.87 & 2166 & 11.90 & 0.1505 & 0.1244 \\
\hline $70-79$ & 8.57 & 19857 & 19.86 & 4102 & 22.54 & 0.2495 & 0.2122 \\
\hline $80-89$ & 4.91 & 23242 & 23.25 & 6813 & 37.44 & 0.3587 & 0.3384 \\
\hline $90+$ & 1.38 & 11909 & 11.91 & 3929 & 21.59 & 0.4155 & 0.3928 \\
\hline Tous & 100 & 99966 & 100 & 18198 & 100 & 0.2143 & 0.1969 \\
\hline
\end{tabular}

\section{Description spatiale de l'épidémie}

L'épidémie présente une distribution spatiale des hospitalisations très inégale durant la période étudiée. Elle s'est propagée dans l'ensemble du pays à bas bruit, avec 84906 
patients en France métropolitaine (cas fermés) ce qui correspond à un taux d'hospitalisation global de 1.31 pour 1000 habitants (soit une incidence de 18.71 pour 100000 personne/semaine), et un taux d'hospitalisation par département en moyenne de 1.14 pour 1000 habitants (médiane à 0.75 ) sur la période, avec un écart-type de 0.95 . Dans certaines régions, la transmission a été beaucoup plus active, comme en Ile-deFrance (moyenne du taux d'hospitalisation à 3.28 pour 1000, soit une incidence de 46.83 pour 100000 personne/semaine), ou en région Grand-Est (moyenne du taux d'hospitalisation à 3.15 pour 1000, soit une incidence de 45.02 pour 100000 personne/ semaine). Le taux d'hospitalisation par département varie de 0.17 pour 1000 habitants (Tarn-et-Garonne) à 4.80 (Territoire de Belfort) (Fig. 3).

Le taux de mortalité hospitalière (non standardisée sur l'âge) présente la même distribution spatiale. Elle varie de 0.01 pour 1000 habitants (Lozère, Tarn-et-Garonne) à 1.21 pour 1000 habitants (Territoire de Belfort), avec une moyenne de 0.24 (médiane $0.14)$ et un écart-type de 0.23 .

Fig. 3. Taux d'hospitalisation (à gauche) et taux de mortalité hospitalière (à droite) pour 1000 habitants pour la Covid-19 en France métropolitaine pour la période du 19 mars au 28 mai 2020

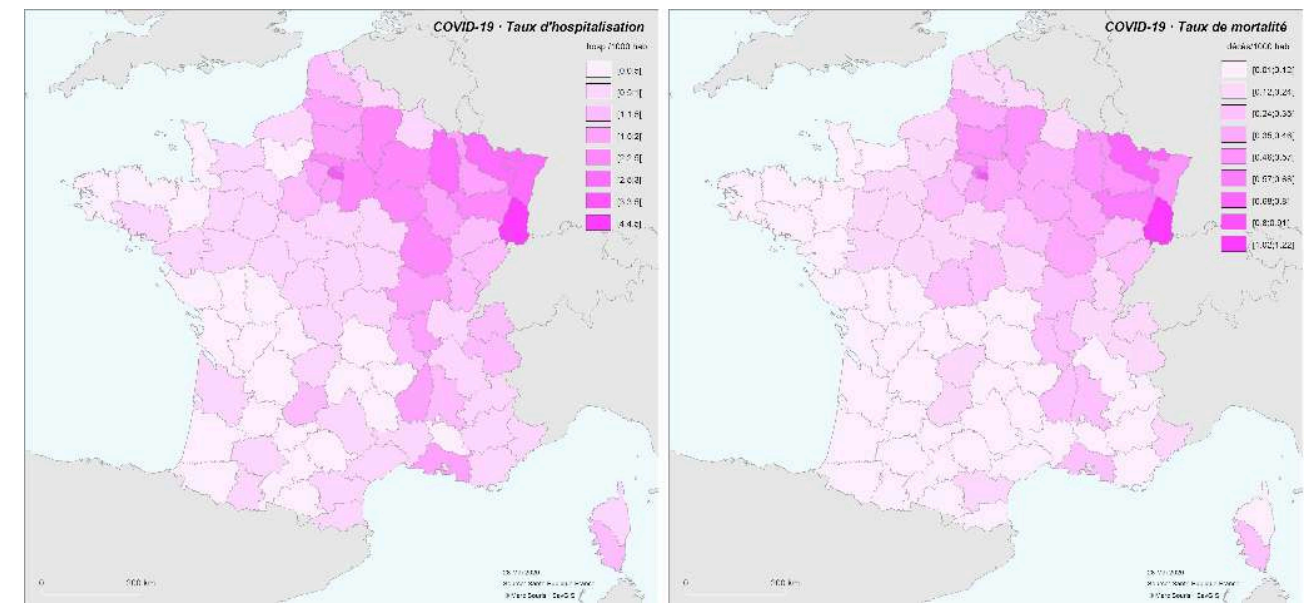

Pendant cette période, le taux de létalité standardisé par département (en excluant les régions Ile-de-France et Grand-Est du calcul des taux de létalité par âge) se situe entre 0.039 (Lozère, SLR=0.20) et 0.44 (Indre, SLR=2.24), avec la moyenne à 0.20 et la médiane à 0.20 , et un écart-type de 0.058 . Le SLR présente une moyenne de 1.02 et un écart-type de 0.30 (Fig. 4). Dans la suite, nous ne considèrerons que les taux de létalité standardisés calculés avec des taux de létalité par âge ne prenant pas en compte les régions Ile-de-France et Grand-Est. 
Fig. 4. Taux de létalité standardisé (à gauche) et ratio de létalité standardisé (à droite) en France métropolitaine pour la période du 19 mars au 28 mai, 2020

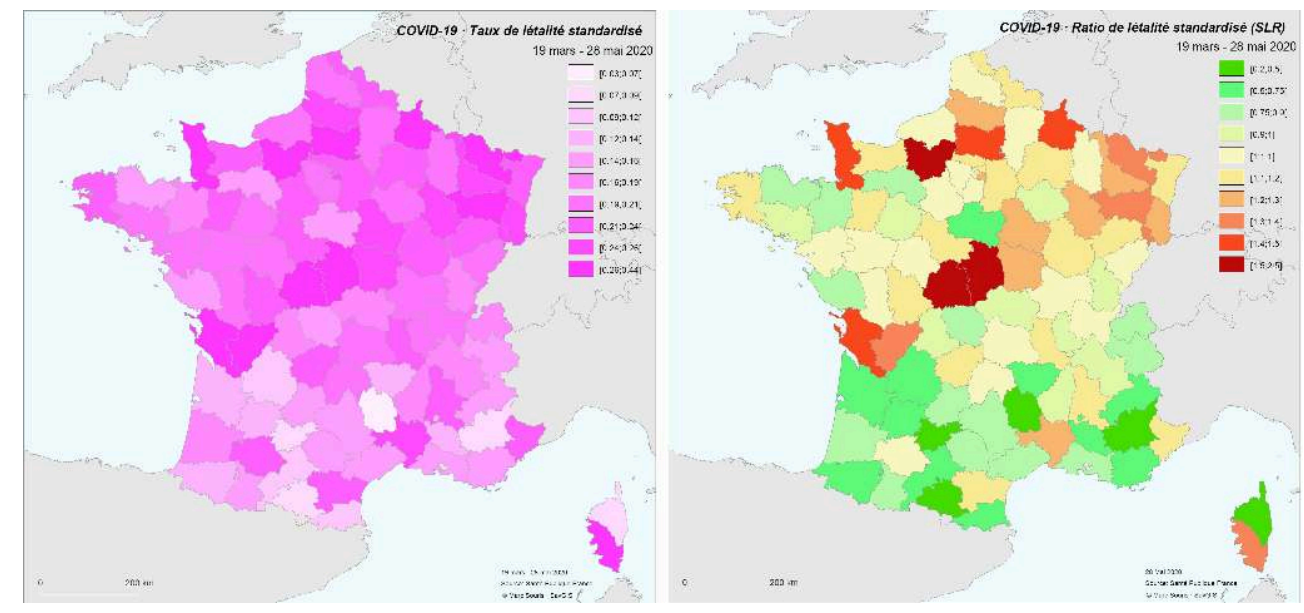

\section{Analyse de l'autocorrélation et de la variabilité spatiale des taux standardisés}

La distribution spatiale du taux d'hospitalisation standardisé présente une autocorrélation spatiale très significative (Moran Index : 3.43, p-value < 10-6), ce qui est plutôt attendu pour une maladie infectieuse (Souris, 2019 ; Pfeiffer \& Robinson, 2008). Le taux de létalité standardisé présente également une autocorrélation spatiale significative (Moran index : 0.27 , p-value $<0.001$ ), ce qui par contre n'est pas attendu. L'analyse des agrégats montre clairement un regroupement des valeurs élevées du taux de létalité dans l'est, le nord, le centre, et des valeurs faibles dans le sud, avec quelques départements ayant des valeurs très élevées, dans le centre (Indre, 0.44, Cher, 0.34) et dans une moindre mesure dans l'ouest (Charente-Maritime, 0.28, Charente, 0.26).

Le test de significativité de Breslow \& Day pour le SLR indique les départements où le SLR est statistiquement significativement différent de 1 , ce qui correspond aux départements où le ratio de létalité standardisé est anormalement élevé (SLR > 1, en rouge) ou anormalement faible (SLR $<1$, en vert). Le seuil de significativité individuel est fixé à 0.05, et pour l'ensemble des départements à 0.001 (Fig. 5).

Fig. 5. Significativité du SLR par département : $\mathrm{p}$-value $<0.05$ (à gauche), p-value $<0.001$ (à droite)

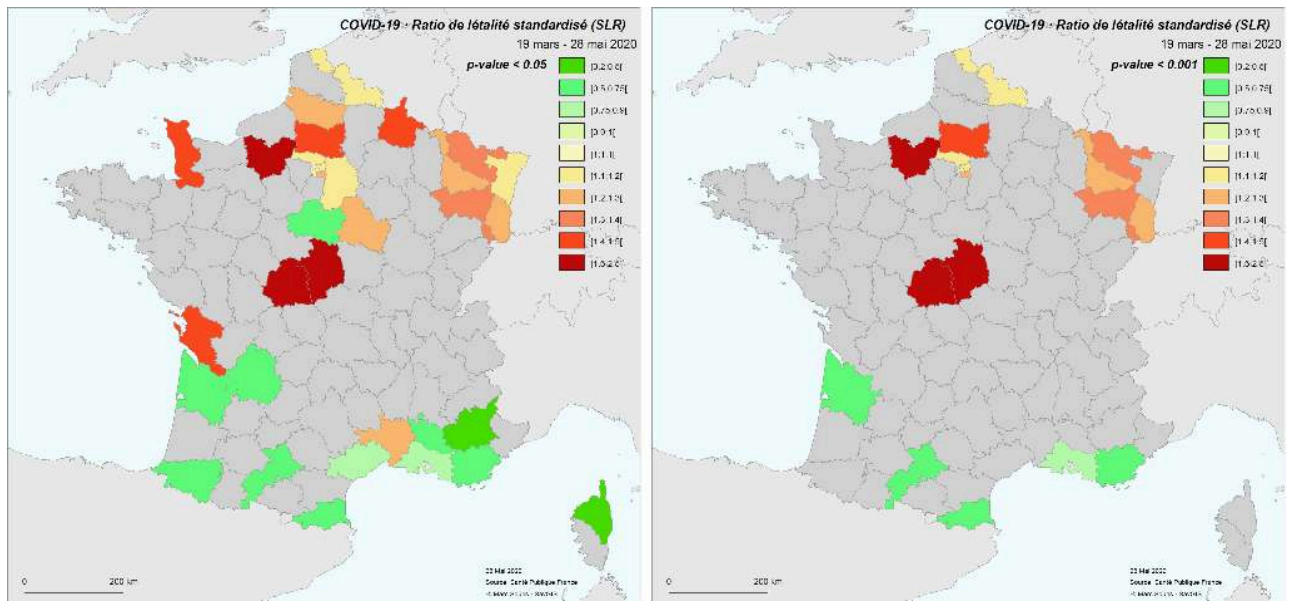




\section{Analyse des facteurs de risque}

Le système de santé en France offre en moyenne 621 lits d'hôpital pour 100000 personnes, et cette capacité hospitalière par habitant est répartie de façon assez uniforme sur l'ensemble du territoire métropolitain (Tableau 4).

Tableau 4 : Nombre de lits d'hôpital pour 100000 personnes, par région en France métropolitaine

\begin{tabular}{|c|c|}
\hline Région & $\begin{array}{c}\text { Nombre de lits } \\
\text { d'hôpital pour } \\
100000 \text { personnes }\end{array}$ \\
\hline France métropolitaine & 621 \\
\hline Île de France & 547 \\
\hline Centre-Val de Loire & 634 \\
\hline Bourgogne-Franche-Comté & 711 \\
\hline Normandie & 621 \\
\hline Hauts-de-France & 634 \\
\hline Grand Est & 642 \\
\hline Pays de la Loire & 569 \\
\hline Bretagne & 651 \\
\hline Nouvelle Aquitaine & 686 \\
\hline Occitanie & 691 \\
\hline Auvergne-Rhône-Alpes & 659 \\
\hline Provence-Alpes-Côte d'Azur & 778 \\
\hline Corse & 678 \\
\hline
\end{tabular}

31 Il n'y a pas de corrélation entre la capacité des hôpitaux (nombre de lits pour 100000 personnes) et le taux de létalité standardisé (indice de Bravais-Pearson $\mathrm{r}=-0,11$ ).

Après standardisation sur l'âge, il n'y a pas de corrélation (indice de Bravais-Pearson $\mathrm{r}=0.14$ ) entre le taux de létalité standardisé et le taux des facteurs de risque par département (ex.; maladies coronariennes, hypertension, maladies respiratoires aiguës, diabète).

Par contre, on constate une corrélation significative entre les taux standardisés d'hospitalisation et la létalité au niveau des départements (Bravais-Pearson index $\mathrm{r}=0.30)$, corrélation qui augmente $(\mathrm{r}=0.42)$ lorsqu'on limite le calcul aux départements dont le taux standardisé d'hospitalisation a dépassé 0.5 pour 1000 . On constate notamment des taux de létalité élevés dans tous les départements qui présentent des taux d'hospitalisations élevés (à part les Bouches-du-Rhône) : pour les 20 départements où les taux d'hospitalisation ont été les plus élevés (essentiellement situés dans l'Est et en Ile-de-France), la moyenne des taux de létalité (0.23) est significativement plus élevée que la moyenne des taux effectuée sur l'ensemble des autres départements (0.19).

34 Le tableau 5 donne le taux de létalité standardisé moyen sur les départements en fonction de leur taux d'hospitalisation standardisé. 
Tableau 5. Taux moyen de létalité standardisé en fonction du taux d'hospitalisation standardisé pendant la première phase de l'épidémie de Covid-19 en France (du 19 mars au 28 mai 2020)

\begin{tabular}{|l|l|l|}
\hline $\begin{array}{c}\text { Taux } \\
\text { d'hospitalisation } \\
\text { standardisé (pour } \\
1000 \text { hab.) }\end{array}$ & $\begin{array}{c}\text { Nombre de } \\
\text { départements }\end{array}$ & $\begin{array}{c}\text { Taux de létalité } \\
\text { standardisé moyen }\end{array}$ \\
\hline$<0.5$ & 30 & 0.183 \\
\hline $0.5-1.0$ & 29 & 0.189 \\
\hline $1.0-1.5$ & 13 & 0.214 \\
\hline $1.5-2.0$ & 7 & 0.240 \\
\hline $2.0-3.0$ & 10 & 0.235 \\
\hline$>3.0$ & 7 & 0.253 \\
\hline
\end{tabular}

Le rapport entre le taux de patients en réanimation et le taux d'hospitalisation (qui donne en principe une indication de la sévérité des malades hospitalisés) montrent une faible corrélation négative ( $\mathrm{r}=-0.22$ ), indiquant une tendance à la baisse du premier lorsque le second (hospitalisations) est élevé. Cette tendance peut être due à la saturation des services de réanimation ou par une baisse des hospitalisations moins sévères pour pouvoir prendre en charge plus de cas sévères en phase de saturation, ce qui aurait pour conséquence d'augmenter la létalité. Néanmoins, dans les deux cas, on ne constate pas de corrélation entre le taux des réanimations et le taux de létalité $(\mathrm{r}=-0.1)$, indiquant que globalement l'intensité du recours à la réanimation n'impacte pas le taux de létalité. Enfin, le taux des réanimations ne présente pas de distribution spatiale correspondant à la distribution spatiale du taux d'hospitalisation (Fig. 6). Il faut néanmoins noter que $73 \%$ des décès à l'hôpital n'ont pas été hospitalisés en réanimation, et notamment les patients les plus âgés, cela principalement pour des raisons médicales et non pas pour des raisons de tension hospitalière (Courtejoie \& Dubost, 2020). 
Fig. 6. Taux de réanimation pendant l'épidémie de Covid-19 en France du 19 mars au 28 mai 2020.

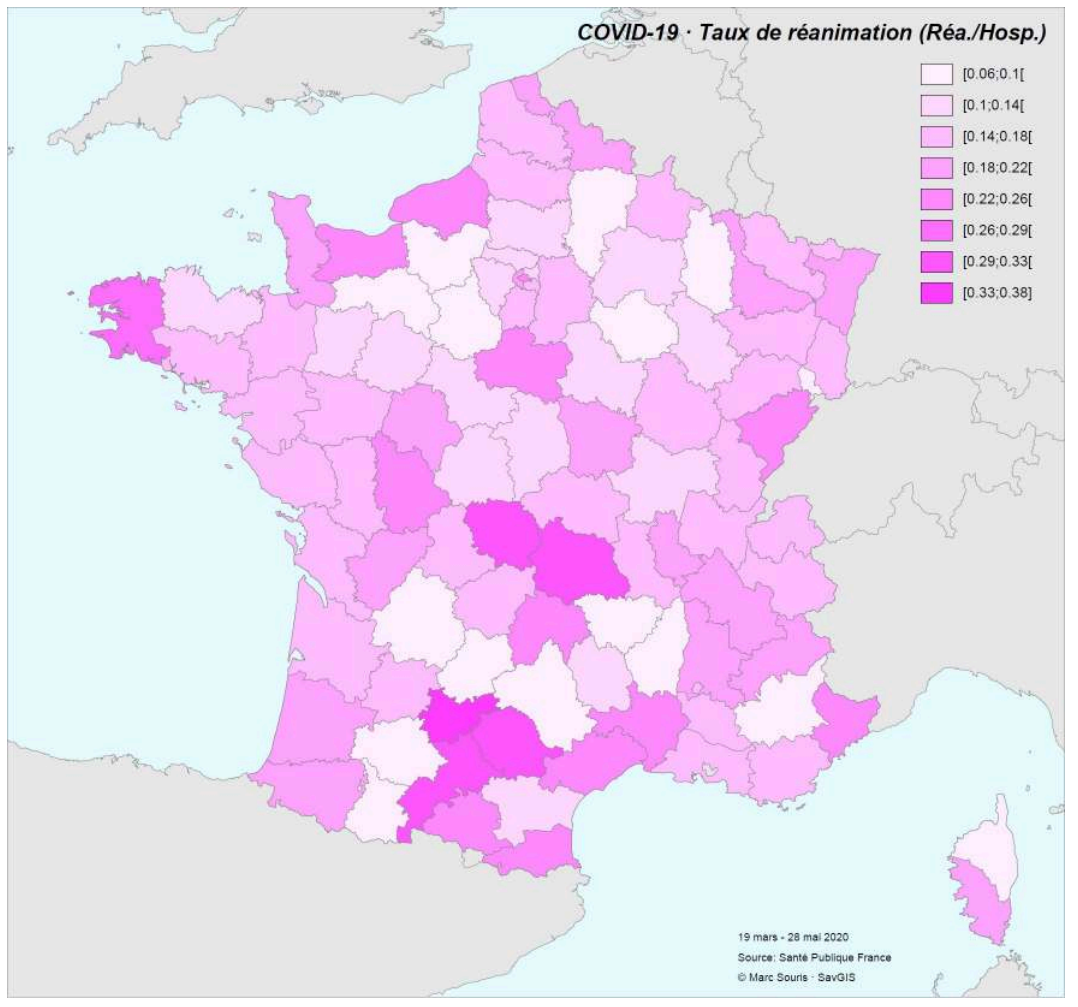

Une typologie combinant les taux d'hospitalisation et les taux de létalité est proposée, après classification en trois classes pour chacun des taux d'après la distribution des valeurs, la classe centrale étant centrée sur la moyenne (pour le taux d'hospitalisation : $<0.90,0.90-1.20,>1.20$, pour la létalité : $<0.18,0.18-0.22,>0.22$ ) de façon à classer les départements en cinq classes (faible-faible, faible-fort, fort-faible, fort-fort, autres) (Fig. 7). 
Fig. 7. Combinaison des taux standardisés d'hospitalisation et de létalité en cinq classes

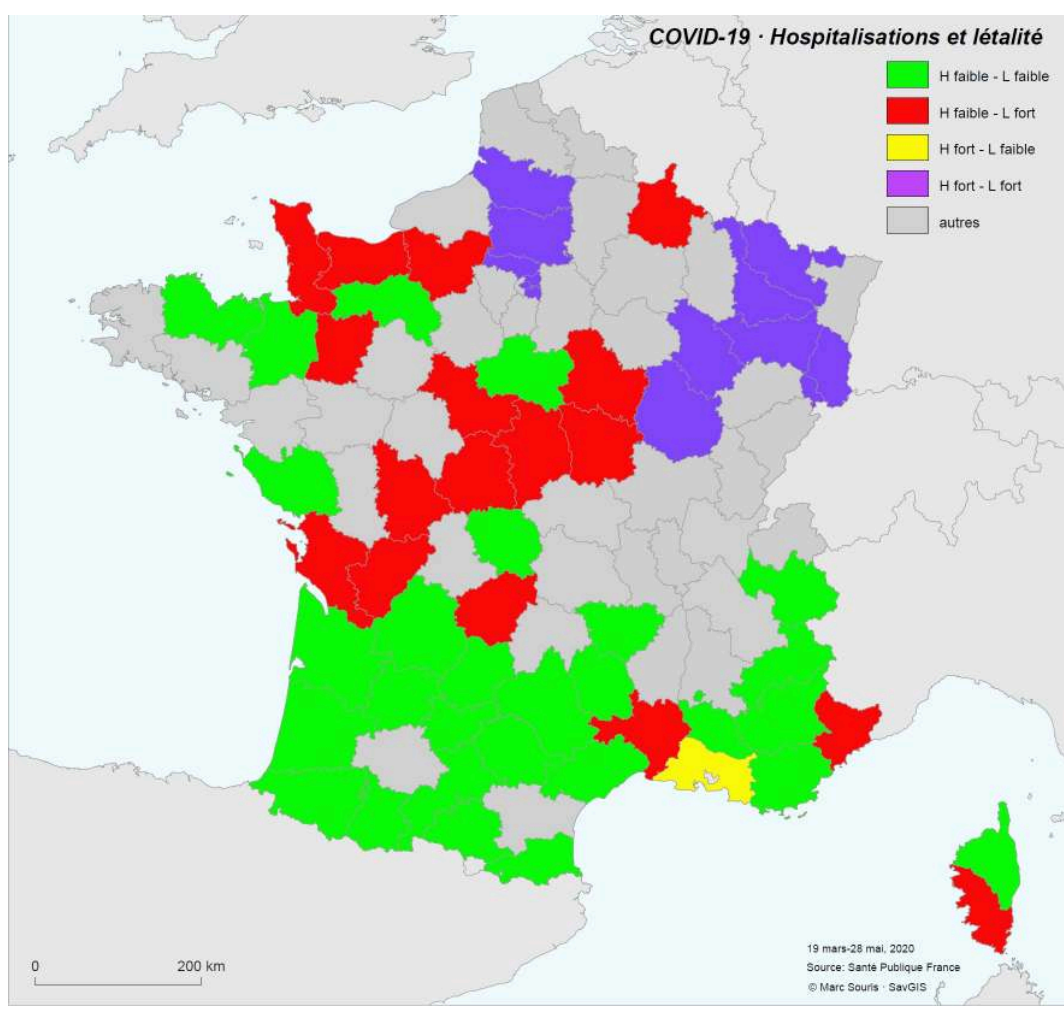

\section{Discussion}

La capacité hospitalière étant répartie de façon assez uniforme sur le territoire métropolitain, on peut supposer que la tension hospitalière due à la Covid-19 dans un département n'est pas liée à la capacité hospitalière mais uniquement à l'incidence de la maladie. Effectivement, on ne trouve pas de corrélation directe entre capacité hospitalière et taux de létalité.

Après classification et combinaison des taux d'hospitalisation et des taux de létalité standardisés comme indiqué ci-dessus, deux profils à risque se dégagent : d'une part, les départements où un fort taux d'hospitalisation est associé à un fort taux de létalité, et d'autre part les départements où un faible taux d'hospitalisation est couplé à un fort taux de létalité.

Le premier profil, correspondant à la classe «fort taux d'hospitalisation-fort taux de létalité ", réunit 13 départements, représente $37 \%$ des patients hospitalisés (39409 sur $106917)$ et $42 \%$ des décès (7840 sur 18633). Ces départements, spatialement regroupés (Indice de Moran $=1.58, \mathrm{p}$-value $<10-6$ ), correspondent aux épicentres de l'épidémie et incluent les zones densément peuplées d'Ile-de-France (densité de population moyenne 26.97 hab./ha). La moyenne du taux de létalité standardisé y est significativement plus élevée (0.25) que dans les autres départements $(0.19)$. Cet excès de mortalité (1140 décès, pour un taux de létalité standardisé de 0.17 correspondant à la moyenne des taux des départements hors des deux profils à risque) résulte vraisemblablement d'une hausse de la létalité due à la surcharge du système hospitalier.

Le second profil, correspondant à la classe « faible taux d'hospitalisation-fort taux de létalité », réunit quinze départements, représente $5.3 \%$ des patients (5666 sur 106917) 
et $5.9 \%$ des décès (1101 sur 18633), avec une moyenne du taux de létalité standardisé significativement élevée (0.268) correspondant néanmoins à un excès de mortalité de faible ampleur (estimé à 138 décès). Ces quinze départements, qui ne sont pas significativement regroupés (indice de Moran $=0.18, \mathrm{p}$-value $=0.13$ ), sont essentiellement situés dans des zones de densité de population significativement plus faible que dans l'ensemble des autres départements ( 0.75 hab./ha contre $6.12 \mathrm{hab} . / \mathrm{ha}$, $\mathrm{p}$-value $=0.02$ ) ou que dans l'autre profil ( $p$-value $=0.03$ ).

Il est possible que la sur-létalité de ce profil soit due à un plus faible ou plus tardif recours aux soins (désert médicaux, faible préparation hospitalière), ce que semble renforcer le plus faible taux d'accessibilité aux médecins généralistes pour les départements de ce profil (3.34 contre 2.99, $\mathrm{p}$-value $=0.03$. Le taux représente le nombre de consultations ou visites accessibles de médecine générale par an et par habitant standardisé) (Fig. 8).

Fig. 8. Indicateur d'accessibilité potentielle localisée (APL). De rouge à vert, de faible à forte accessibilité

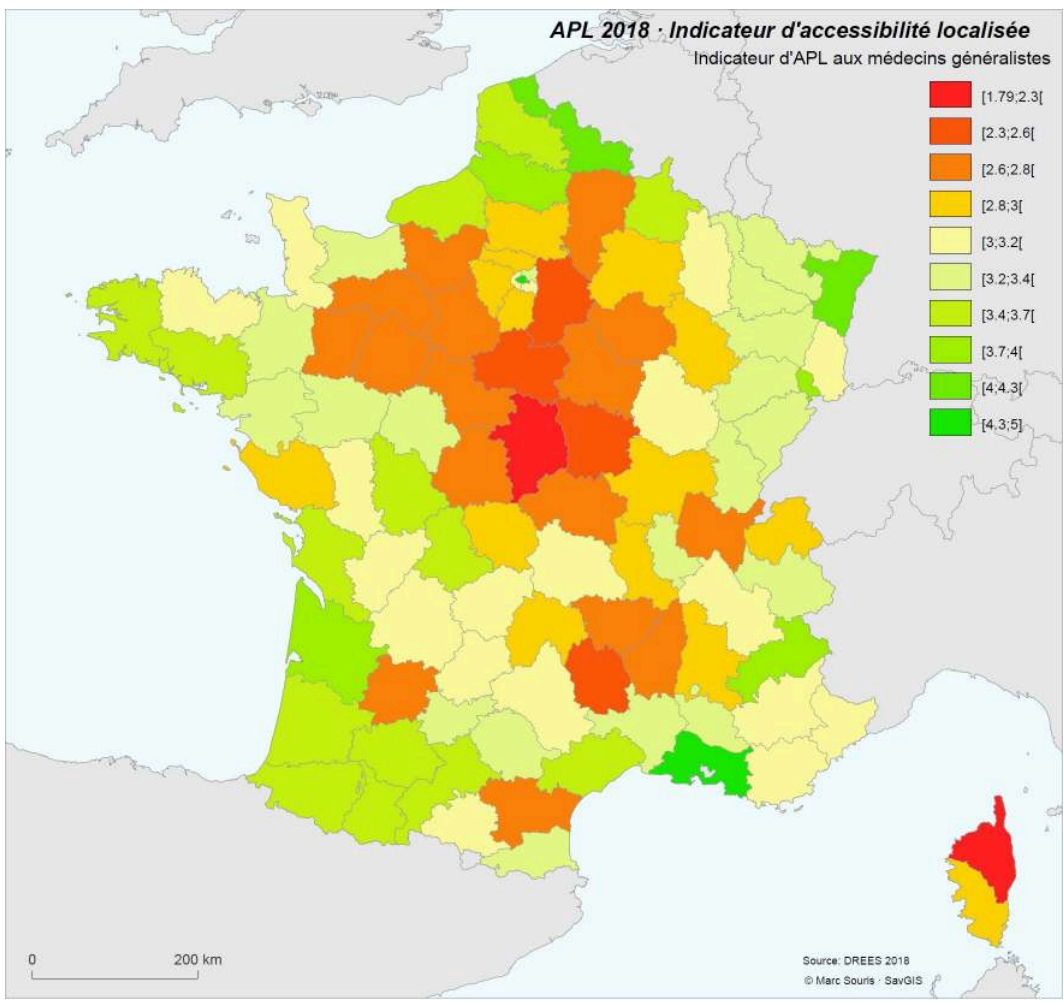

On note aussi que certains départements du sud de la France présentent à la fois un très faible taux d'hospitalisation et un très faible taux de létalité (la Gironde, la Dordogne, le Gers, les Pyrénées Orientales). Enfin, on note également le cas unique des Bouches du Rhône, qui apparait avec un fort taux d'hospitalisation (1.96 pour 1000, pour une moyenne nationale de 1.27) et un taux de létalité standardisé plutôt faible (0.14), ainsi qu'un très bon taux d'accessibilité. Cette situation pourrait être due à une politique médicale de dépistage plus active et d'hospitalisation plus précoce des infectés dans le département, et notamment dans l'aire métropolitaine de Marseille. La valeur élevée du taux d'hospitalisation résulterait alors plus d'une prise en charge des infectés non sévères que d'une circulation plus active du virus dans la région, comme semblerait le montrer les taux d'hospitalisation plus faibles des départements voisins ( 0.9 pour le 
Var, 0.56 pour le Vaucluse, 0.52 pour le Gars, 0.86 pour les Alpes-de-Haute-Provence, 0.81 pour les Alpes-Maritimes, 0.68 pour l'Hérault). Cependant, le profil de sévérité des patients (exprimé par le taux de réanimation) n'est pas particulièrement faible dans les Bouches-Du-Rhône (0.163, pour une moyenne de 0.156 et un écart-type de 0.06 sur l'ensemble des départements), et cette politique d'hospitalisation n'est donc pas avérée comme facteur premier du faible taux de létalité observé dans ce département.

Au niveau international, fin mai 2020 les données de l'OMS sur 219 pays (https:// covid19.who.int/) indiquaient un taux de létalité moyen de 0.041 (médiane à 0.029 , écart-type de 0.045) avec des valeurs comprises entre 0.31 (Nicaragua) et 0 (pour les pays n'ayant pas déclaré de décès au 28 mai 2020). Même si l'on ne considère que la moyenne de létalité calculée sur les départements français présentant les plus faibles taux d'hospitalisation (ne provoquant donc pas une possible mise en tension du système hospitalier), cette moyenne est toujours restée très significativement supérieure à la moyenne mondiale (et aux taux de la plupart des pays d'Europe, comme l'Espagne, 11.73, la Grèce, 5.52, l'Allemagne, 4.28, etc.) (tableau 1). Nous constatons que la variabilité spatiale interne à la France, même si elle a été importante au cours de la période étudiée (écart-type 0.057), ne permet pas d'expliquer les importantes différences dans les taux de létalité entre pays. Ces différences de létalité ne peuvent être expliquées ni par les différences de morbidité ni par les différences de traitement des patients, compte-tenu de la bonne qualité du système de santé et de soins en France (Tableau 1 et 4). On peut en conclure que les différences résultent essentiellement d'une différence d'enregistrement des cas et/ou des décès. Ces différences de comptage peuvent être dues à la politique d'hospitalisation et de dépistage propre à chaque pays (ex. capacité ou volonté d'hospitaliser plus de formes non sévères), à des différences dans la définition du cas, ou à une qualité limitée du système de détection et de report des cas et des décès. Cette conclusion est renforcée par les études sur la surmortalité (par rapport à la moyenne de la mortalité des années antérieures sur la même période) qui montrent que, dans la plupart des pays, cette surmortalité n'est pas totalement expliquée par les décès officiellement attribués à la Covid-19, alors que la France montre un léger excès de décès au Covid-19 déclarés (Le Temps, 2020).

\section{Conclusion}

Cette étude montre que les taux de létalité élevés observés en France dans certains départements pendant la première vague de l'épidémie de Covid-19 pourraient être liés à deux phénomènes : d'une part au niveau de morbidité dans le département, donc à la possible tension des systèmes de soins pendant la phase aigüe de l'épidémie et d'autre part, lorsque le taux d'hospitalisation est resté faible, à l'accès aux soins dans des départements de faible densité de population.

Néanmoins, l'augmentation du taux de létalité standardisé dû aux situations exceptionnelles lors du pic épidémique ne peut expliquer l'ampleur de la différence constatée entre le taux de létalité moyen en France et ceux reportés sur l'ensemble des pays par les organisations ou les sites d'information internationales (WHO, Wordometer, etc.). Ces différences proviendraient très vraisemblablement de la déclaration inégale des cas et des décès selon les pays, et non de la prise en charge des patients. 


\section{BIBLIOGRAPHIE}

Baud D, Qi X, Nielsen-Saines K, Musso D, Pomar L, Favre G, 2020. Real estimates of mortality following Covid-19 infection. Lancet Infect Dis. https://doi.org/10.1016/S1473-3099(20)30195-X Bouyer J, Hémon D, Cordier S, Derriennic F, Stûcker I, Stengel B, Clavel J, 1993. Epidémiologie, principes et méthodes quantitatives. Inserm.

Breslow NE and Day NE, 1987. Statistical methods in cancer research, vol. I \& II. WHO, International Agency for Reseach on Cancer.

Collier L, J. Oxford, 2016. Human Virology. Oxford University Press, 2016.

Courtejoie N, Dubost CL, 2020. Parcours hospitalier des patients atteints de la Covid-19 lors de la première vague de l'épidémie. Les Dossiers de la DREES, $n^{\circ} 67$, DREES.

Hurraux JM, Nicolas JC, Agut H, Peigne-Lafeuille H, 2003. Traité de virologie humaine. Estem/ AUF.

Le Temps, 2020. L'impact du Covid-19 sous-estimé dans de nombreux pays. Available online: https://labs.letemps.ch/interactive/2020/surmortalite-et-covid-19/

Miller RG, 1996. Simultaneous Statistical Inference. Springer Verlag.

Morteza AK, Fakher R, 2020. Cross-Country Comparison of Case Fatality Rates of Covid-19/SARSCOV-2. Osong Public Health and Research Perspectives; 11(2): 74-80.

Pfeiffer D, Robinson T, Stevenson M, Stevens K, Rogers D, Clements A, 2008. Spatial Analysis in Epidemiology. Oxford University Press.

Porta M, 2008. A dictionary of Epidemiology. 5th Ed. Oxford University Press, Oxford.

Rinaldia G, Paradisiba M, 2020. An empirical estimate of the infection fatality rate of Covid-19 from the first Italian outbreak. Harvard Economics Department.

Salie H, Tran-Kiem C, Lefrancq N, Courtejoie N, Bosetti P, et all, 2020. Estimating the burden of SARS-CoV-2 in France. Science 13 May 2020. DOI: 10.1126/science.abc3517

Saluzzo JF, Vidal P, Gonzalez JP, 2004. Les virus émergents. IRD Edition.

Souris M, 2019. Epidemiology and Geography. Principles, methods and tools of spatial analysis. Wiley-ISTE, London.

Spychalski P, Blazynska-Spychalska A, Kobiela J, 2020. Estimating case fatality rates of Covid-19. The Lancet, doi: https://doi.org/10.1016/S1473-3099(20)30246-2.

\section{RÉSUMÉS}

L'objectif de cet article est d'analyser les variations spatiales de la létalité hospitalière due à la Covid-19 en France métropolitaine pendant la première vague de l'épidémie.

Les résultats montrent une corrélation positive significative entre niveau de morbidité et taux de létalité. Cependant, l'ampleur de cette augmentation ne peut expliquer l'ampleur des variations des taux reportés par pays qui ne peuvent provenir que des systèmes de déclaration des cas et des décès, très inégaux selon les pays. 
En conclusion, il semble que la létalité élevée observée dans certains départements soit essentiellement due à la surcharge du système de soins dans les départements sous tension hospitalière. Les départements présentant un faible taux d'hospitalisation mais une létalité élevée se trouvent essentiellement dans des zones de faible densité de population, et ce phénomène pourrait être liée à des problèmes d'accès ou de recours aux soins.

INDEX

Mots-clés : Covid-19, létalité, standardisation, analyse spatiale, SLR

\section{AUTEURS}

\section{MARC SOURIS}

UMR Unité des Virus Émergents (UVE Aix-Marseille Univ-IRD 190-Inserm 1207-IHU Méditerranée Infection), Marseille 13005 France

\section{JEAN-PAUL GONZALEZ}

School of Medicine, Georgetown University, Department of Microbiology \& Immunology, Washington, DC 20057 USA. Centaurus Biotech LLC 